\title{
Electron Spin Resonance and Atomic Force Microscopy Study on Gadolinium Doped Ceria
}

\author{
Cesare Oliva, ${ }^{1,2}$ Francesco Orsini, ${ }^{3}$ Serena Cappelli, ${ }^{1}$ Paolo Arosio, ${ }^{3}$ Mattia Allieta, \\ Mauro Coduri, ${ }^{4}$ and Marco Scavini ${ }^{1,2}$ \\ ${ }^{1}$ Dipartimento di Chimica, Università di Milano, Via C. Golgi 19, 20133 Milano, Italy \\ ${ }^{2}$ Istituto di Scienze e Tecnologie Molecolari (ISTM), Consiglio Nazionale delle Ricerche (CNR), 20133 Milano, Italy \\ ${ }^{3}$ Dipartimento di Fisica, Università di Milano, Via Celoria 16, 20133 Milano, Italy \\ ${ }^{4}$ Istituto per l'Energia e le Interfasi (IENI), Consiglio Nazionale delle Ricerche (CNR), Corso Promessi Sposi 29, 23900 Lecco, Italy
}

Correspondence should be addressed to Marco Scavini; marco.scavini@unimi.it

Received 31 October 2014; Accepted 12 January 2015

Academic Editor: Stephen Cooke

Copyright (C) 2015 Cesare Oliva et al. This is an open access article distributed under the Creative Commons Attribution License, which permits unrestricted use, distribution, and reproduction in any medium, provided the original work is properly cited.

\begin{abstract}
A combined electron spin resonance (ESR) and atomic force microscopy (AFM) study on $\mathrm{Ce}_{1-x} \mathrm{Gd}_{x} \mathrm{O}_{2-x / 2}$ samples is here presented, aimed at investigating the evolution of the ESR spectral shape as a function of $x$ in a wide composition range. At low $x(=0.02)$, the spectrum is composed of features at $g_{\mathrm{eff}} \approx 2 ; 2.8 ; 6$. With increasing $x$, this pattern merges into a single $\left(g_{\mathrm{eff}} \approx 2\right)$ broad ESR curve, which assumes a Dysonian-shaped profile at $x \geq 0.5$, whereas, at these $x$ values, AFM measurements show an increasing surface roughness. It is suggested that the last could cause the formation of surface polaritons at the origin of the particular ESR spectral profile observed at these high Gd doping levels.
\end{abstract}

\section{Introduction}

Gadolinium-doped ceria $\mathrm{Ce}_{1-x} \mathrm{Gd}_{x} \mathrm{O}_{2-x / 2}$ are considered one of the most promising electrolytes for many potential practical applications such as solid oxide fuel cells (SOFCs) [1]. In particular, $\mathrm{Ce}_{1-x} \mathrm{Gd}_{x} \mathrm{O}_{2-x / 2}$ showed ionic conductivity higher than conventional Yttria-Stabilized-Zirconia-based electrolytes and were able to operate at lower $\left(500-700^{\circ} \mathrm{C}\right)$ temperatures $[1,2]$. However, the deep understanding of the phenomena which separately contribute to ionic and to electronic conductivity mechanisms is far from trivial because of an intricate interplay between the structural effect induced by doping and the transport properties of ceria.

Neutron and synchrotron radiation powder diffraction data, analyzed both in the reciprocal and real space, have supplied important information on the local, mesoscopic, and average structure, as revealed by some of the present authors for $\mathrm{Ce}_{1-x} \mathrm{Gd}_{x} \mathrm{O}_{2-x / 2}[3,4]$ and for other rare earth (RE) doped ceria samples [5-9]. It was shown that nanoscopic compositional fluctuations exist in doped ceria, but $\mathrm{Ce}$ and dopant ions tend to maintain the structure of the parent $\mathrm{CeO}_{2}$ and rare earth sesquioxides $\left(\mathrm{RE}_{2} \mathrm{O}_{3}\right)$. Ce and $\mathrm{RE}$ rich zones can extend over several nanometres, depending on samples composition and RE dopant $[6,9]$.

Due to the paramagnetic nature of $\mathrm{Gd}^{3+}$ ions, also the electron spin resonance (ESR) is considered a suitable technique to study the nature of these systems. In particular, the ESR line-shape of Gd-containing samples has supplied details of electronic structure of Gd based magnetoresistive perovskites [10].

In $\mathrm{Ce}_{1-x} \mathrm{Gd}_{x} \mathrm{O}_{2-x / 2}$ compounds, the interplay between crystal structure and magnetic properties and sample performances has been already examined by us elsewhere $[11,12]$.

In the present paper we would like to focus the attention on some problems encountered during these investigations and concerning the ESR line-shape and its dependence on the $x$ gadolinium doping level (Figure 1). Indeed, the ESR line changes markedly with $x$. In particular, a single ESR line appears at $x=0.125$, broadening with further $x$ increasing. Two different phenomena could account for the last observation, namely, (a) a shortening of the spinspin relaxation time $T_{2}$ due to increasing spin-spin random 


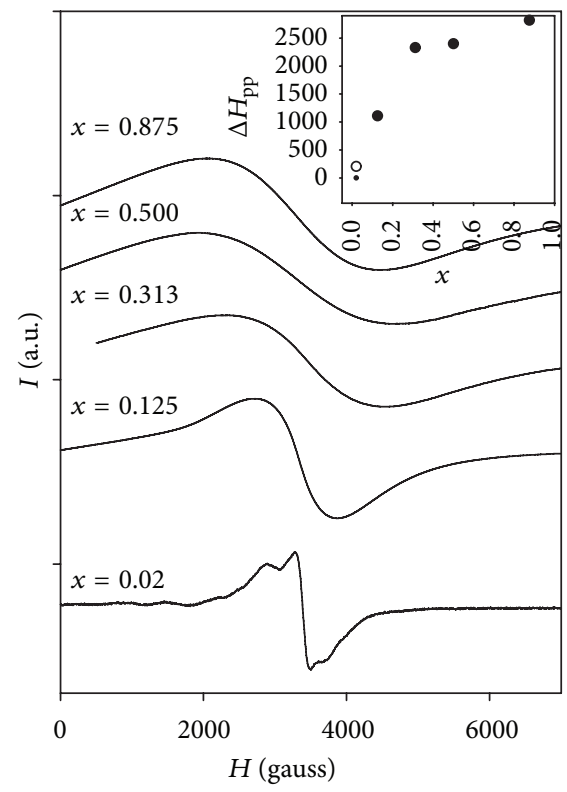

FIGURE 1: ESR spectra at room $T$. The peak-to-peak linewidth $\left(\Delta H_{\mathrm{pp}}\right)$ values for the same samples are reported in the inset (black circles). The white circle refers to $\Delta H_{\mathrm{pp}}$ of the resonance line at $g_{\mathrm{eff}} \approx 2$ for the $x=0.02$ sample.

interactions and (b) the increasing of the internal magnetic field $H_{\mathrm{a}}$ intensity due to formation of ferromagnetic domains. Phenomenon (a) would act on both the lobes of the firstderivative ESR pattern taking away one from the other at the same extent. Phenomenon (b) would act on its left lobe only, moving it towards lower magnetic field values. Furthermore, $\mathrm{Gd}^{3+}$ substitution for $\mathrm{Ce}^{4+}$ means the introduction both of a negative charge and of a magnetic ion into the lattice. Therefore, both charge and magnetic effects could be at the origin of the ESR line changes with $x$. We can conclude that the correct interpretation of the observed ESR line broadening is quite intriguing, because many different phenomena can contribute to it.

The situation simplifies at higher $x$ values when a single mechanism seems to prevail to form the spectral profile. Indeed, we will show that at $x \geq 0.5$ the ESR pattern assumes the shape of a single Dysonian line. This is not new in the literature and it was found also by us in different systems, like $\mathrm{LaMnO}_{3+\delta}$, [13] and $\mathrm{GdBaCo}_{2} \mathrm{O}_{5+\delta}[10,14]$. In those cases, that spectral profile was attributed to the electron mobility. However, it has been also reported [11] that electrons in $\mathrm{Ce}_{1-x} \mathrm{Gd}_{x} \mathrm{O}_{2-x / 2}$ have scarce or null mobility at $x \geq 0.2$. Therefore, combining ESR and atomic force microscopy (AFM) measurements on selected samples, a different explanation is here suggested for the Dysonian profile revealed by the ESR spectra at $x \geq 0.5$.

\section{Materials and Methods}

2.1. Sample Preparation and Characterisation. Microcrystalline $\mathrm{Ce}_{1-x} \mathrm{Gd}_{x} \mathrm{O}_{2-x / 2}$ samples with $\mathrm{Gd}$ concentrations $x=$ $0.02,0.125,0.313,0.500$, and 0.875 were prepared with the Pechini sol-gel method [15]. For each gram of desired product, cerium nitrate, $\mathrm{Ce}\left(\mathrm{NO}_{3}\right)_{3} \cdot 6 \mathrm{H}_{2} \mathrm{O}$ (Aldrich 99\%), and gadolinium nitrate $\mathrm{Gd}\left(\mathrm{NO}_{3}\right)_{3} \cdot 6 \mathrm{H}_{2} \mathrm{O}$ (Aldrich 99.9\%) were dissolved into about $150 \mathrm{~mL}$ of water in the wanted $\mathrm{Gd} / \mathrm{Ce}$ stoichiometric ratio. 24 grams of citric acid (Aldrich 99\%) and $6 \mathrm{~mL}$ of ethylene glycol (Aldrich $\geq 99 \%$ ) were added as polymerization agents. Then the solution was heated and stirred to remove water thus forming a gel. The gel was cast into a ceramic crucible and burned in an ashing furnace (Nabertherm), heated with a heating ramp of about $3 \mathrm{~K} / \mathrm{min}$ up to $773 \mathrm{~K}$, and then held at the same temperature for 3 hours. The powder was then pressed into pellets and fired at $1173 \mathrm{~K}$ for 72 hours in air.

The structure of our samples has been investigated by means of synchrotron radiation powder diffraction analysis. The experimental patterns and the structural analysis are reported elsewhere [4]. All the samples were single phase with $\mathrm{CeO}_{2}$ type structure (space group $F m-3 m$ ) for $x=0.02$ and $x=0.125$ and with $\mathrm{Gd}_{2} \mathrm{O}_{3}$ type structure (space group Ia-3) for $x=0.313,0.500$, and 0.875 .

2.2. ESR Measurements. ESR measurements on the $x=0.02$, $0.125,0.313,0.500$, and 0.875 samples were performed by a Bruker ELEXSYS spectrometer equipped with an ER4102ST standard rectangular cavity at $X$ band $(9.4 \mathrm{GHz})$ frequency at room temperature. Powdered sample was placed into quartz tube and the derivative $d P / d H$ of power $P$ absorbed was recorded as a function of the static magnetic field $H$.

2.3. AFM Imaging. Samples with $x=0.125,0.313,0.5$, and 0.875 were dispersed in isopropanol and sonicated for about $4 \mathrm{~h}$. For AFM imaging, a $5 \mu \mathrm{L}$ drop was pipetted on a freshly cleaved mica disk and after $\sim 30 \mathrm{~s}$ the sample was blown dry with nitrogen gas. AFM imaging was performed using a Nanoscope Multimode IIId system (Bruker, Santa Barbara, CA, USA) operating in air in tapping mode. AFM images were collected using the RMS amplitude of the cantilever as the feedback signal for the vertical sample position. The RMS free amplitude of the cantilever was approximately $15 \mathrm{~nm}$ and the relative set-point above $95 \%$ of the free amplitude. Rectangular silicon probes with nominal spring constant around $2.5 \mathrm{~N} / \mathrm{m}$ (NSG01, NT-MDT, Russia) and cantilever length of $120 \mu \mathrm{m}$ were used. The cantilever resonance frequency was about $130 \mathrm{kHz}$. The mica support (Ted Pella, CA, USA) was glued to a metal disk that was magnetically fixed to the AFM sample holder. Images were recorded at $\sim 1 \mathrm{~Hz}$ line rate and a resolution of $512 \times 512$ pixels per image was chosen. AFM images were subject to a line-by-line subtraction of linear background to eliminate sample tilt from the images and correct for stepwise changes between individual scan lines.

\section{Experimental Results}

3.1. The ESR Spectra. All the ESR spectra are reported in Figure 1. At the lowest $(x=0.02) \mathrm{Gd}^{3+}$ concentration the spectrum is composed of features at $g_{\text {eff }} \approx 2,2.8$, and 6 in agreement with what is reported in the literature [16] with diluted solid solutions of this kind. This pattern merges into 


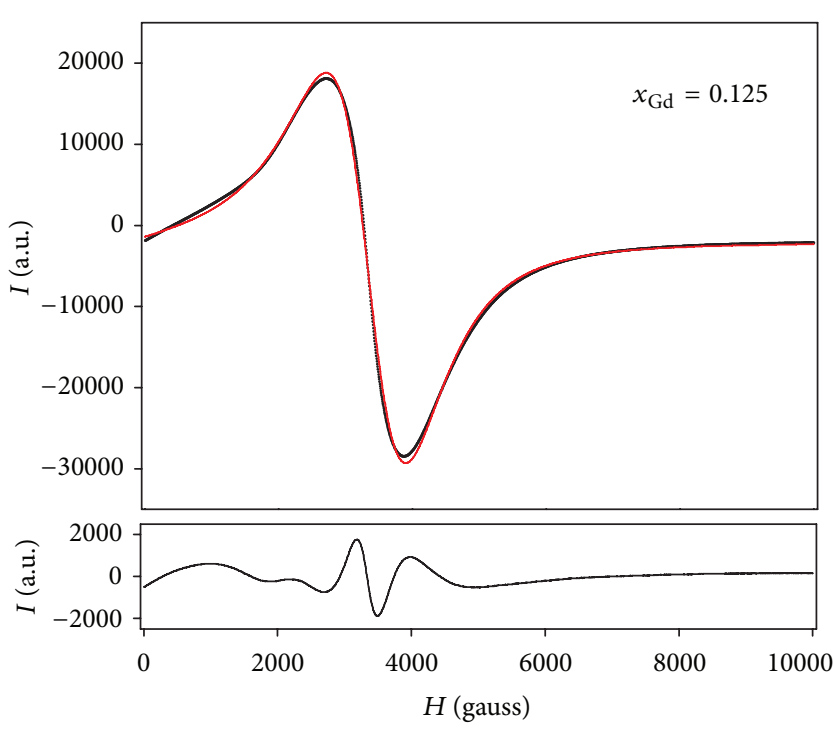

(a)

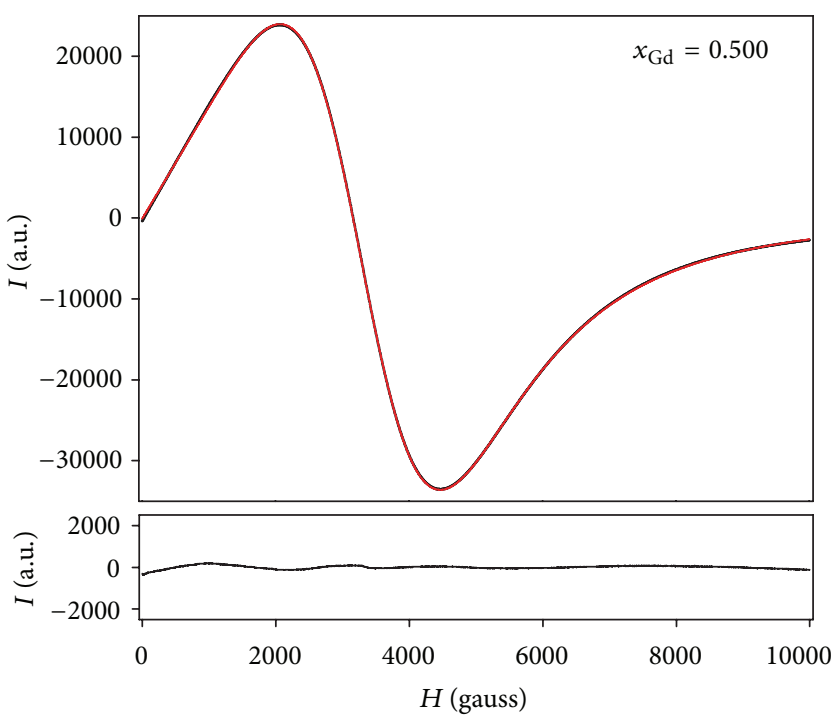

(c)

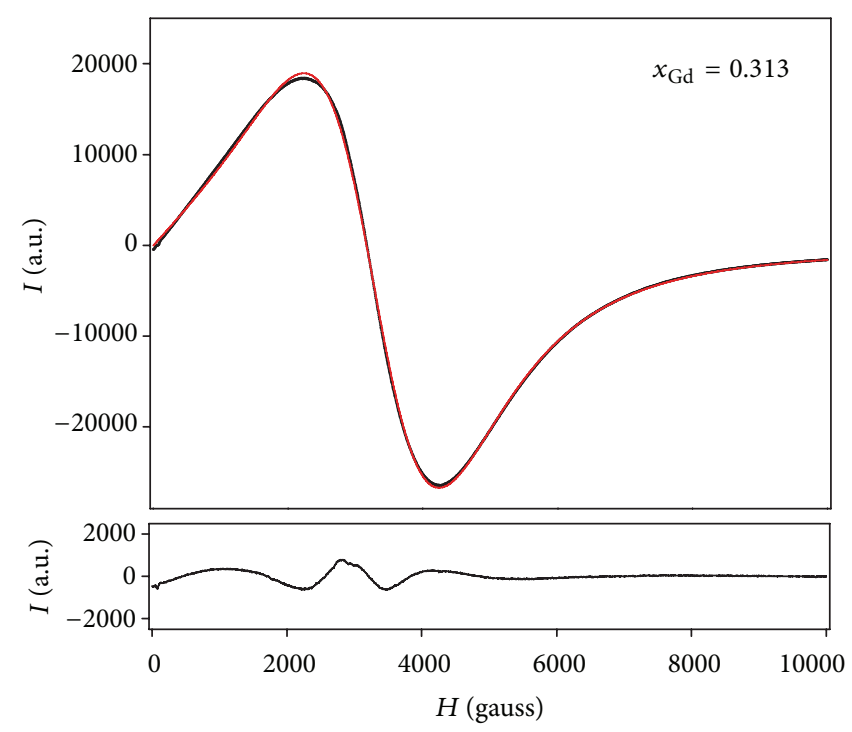

(b)

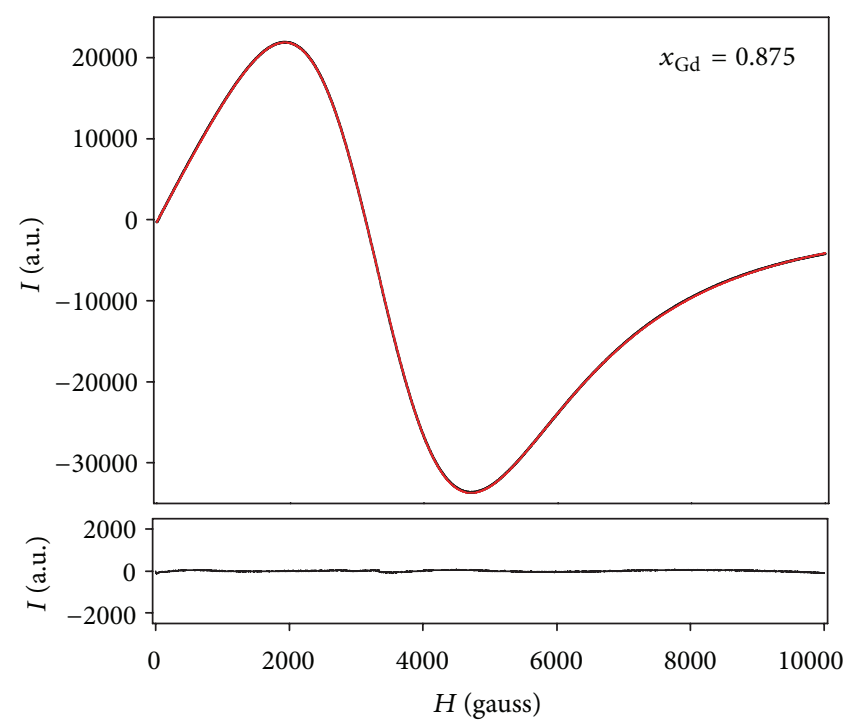

(d)

Figure 2: Dysonian fits of ESR lines for samples with $x_{\mathrm{Gd}}=0.125,0.313,0.50$, and 0.875 . Experimental spectra are compared to the fitted curves (red on line). The differences are at the bottom.

a single $\left(g_{\text {eff }} \cong 2\right)$ broad resonance line at $x \geq 0.125$, with peak-to-peak linewidth $\left(\Delta H_{\mathrm{pp}}\right)$ increasing with gadolinium concentration (see inset of Figure 1). This trend is steeper in the $\mathrm{CeO}_{2}$-like solid solution zone than in the $\mathrm{Gd}_{2} \mathrm{O}_{3}$ like one. Attempts to fit the spectral profile by Lorentzian, Gaussian, and Dysonian functions were carried on for all the experimental spectra at $x \geq 0.125$. The best fit was always obtained by a Dysonian function according to

$$
\frac{d P}{d H} \propto \frac{d}{d H}\left(\frac{\Delta H+\alpha\left(H-H_{0}\right)}{\left(H-H_{0}\right)^{2}+\Delta H^{2}}+\frac{\Delta H+\alpha\left(H+H_{0}\right)}{\left(H+H_{0}\right)^{2}+\Delta H^{2}}\right) .
$$

$\Delta H$ is the linewidth, $\alpha$ is dispersion-to-absorption ratio, and $H_{0}$ is the resonance field. The profile fitting performed by using (1) is reported in Figure 2, for samples with $x \geq 0.125$. However, only at $x \geq 0.5$, a single analytical curve of this kind was able to describe the experimental data with good accuracy.

3.2. AFM Imaging. AFM topography images of samples with $x=0.125$ and 0.875 , absorbed on a freshly cleaved mica disk and visualized in air operating in tapping mode, have been reported in Figure 3, as an example. The two surfaces appear quite different from each other. Indeed, the $x=0.125$ sample has a more squared and planar appearance whereas the surface of the sample with the higher $x$ is rougher with rounded features.

In addition, AFM imaging allowed quantitatively the characterization of the surface morphology of all the samples. 


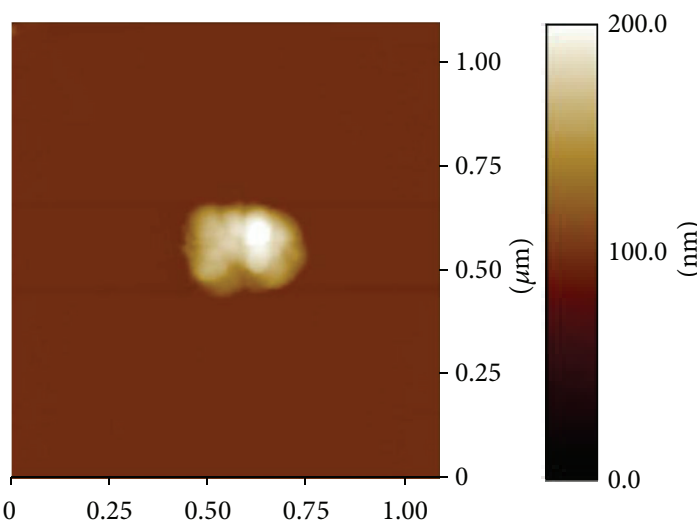

(a)

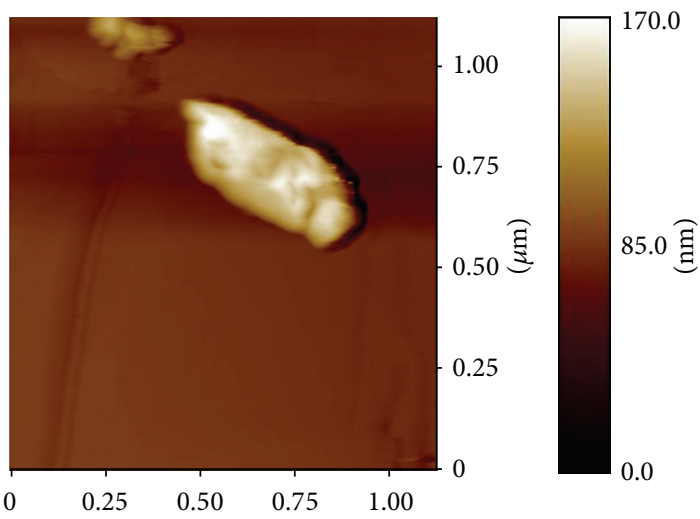

(b)

FIGURE 3: AFM topography images of 0.125 (a) and 0.875 (b) samples absorbed on a freshly cleaved mica disk and visualized in air operating in tapping mode.

TABLE 1: rms surface roughness values measured on $30 \times 30 \mathrm{~nm}^{2}$ areas and obtained analysing more than 20 AFM topography images.

\begin{tabular}{lccc}
\hline \multirow{2}{*}{ Sample } & \multicolumn{3}{c}{ rms surface roughness } \\
& Mean (nm) & SE (nm) & $n$ \\
\hline 0.125 & 1.11 & 0.07 & 27 \\
0.313 & 1.41 & 0.06 & 26 \\
0.5 & 1.81 & 0.06 & 26 \\
0.875 & 2.22 & 0.14 & 27 \\
\hline
\end{tabular}

In particular, the sample surface roughness has been calculated as root-mean-square (rms) data using the following equation:

$$
\mathrm{rms}_{x y}=\sqrt{\sum_{x, y=1}^{N} \frac{\left(Z_{x, y}-Z_{\text {average }}\right)^{2}}{N^{2}}},
$$

where $Z_{\text {average }}$ is the average $Z$ value within the examined area, $Z_{x, y}$ is the local $Z$ value, and $N$ indicates the number of points within the area.

The rms roughness experimental results, measured on $30 \times 30 \mathrm{~nm}^{2}$ areas and obtained analysing about $20 \mathrm{AFM}$ topography images taken from several different samples, have been reported in Table 1.

It is worth noting that the surface of all the samples appears to be quite flat with corrugations of 1-2 $\mathrm{nm}$. Interestingly, the rms surface roughness values are increasing with $x$. In fact, while the $x=0.125$ sample has a rms surface roughness, on average, of $1.11 \mathrm{~nm}$, the sample with the highest $x$ value shows a surface corrugation, on average, of $2.22 \mathrm{~nm}$. A different trend can be observed analysing the distribution of the rms surface roughness values as reported in Figure 4. In particular, the histograms show a narrow and peaked distribution for the samples with low $x$, whereas a broader distribution is exhibited by the samples with the highest Gd content.

\section{Discussion and Conclusion}

The ESR pattern of the $x=0.02$ sample (see Figure 1 ) is very similar to the "U-type" spectra observed in various disordered systems at low concentration of $\mathrm{Gd}^{3+}$ doping. In particular, Cugunov and Kliava attributed its $g_{\text {eff }} \approx 6$ contribution to $\mathrm{Gd}^{3+}$ ions in rhombic or lower crystal-field symmetry in glasses and the broad $g_{\text {eff }} \approx 2$ Gaussianshaped contribution to clustered $\mathrm{Gd}^{3+}$ ions [17]. However, these authors were not able to explain why the former feature disappeared at higher microwave frequencies. Koopmans et al. [18] observed analogous ESR features with $\mathrm{Gd}^{3+}$ doping Ti-based perovskites and assigned them to a superposition of a large number of well-defined spectra. Furthermore, they interpreted the disappearance of the $g_{\text {eff }} \approx 6$ line with increasing microwave frequency, reported elsewhere also by us [19], attributing that feature to a "forbidden" transition with low probability that is reduced to zero when the Zeeman term is larger than the crystal field term. In other studies, features observed at $g_{\text {eff }} \approx 2,2.8$, and 6 were assigned to transitions within the multiplet of energy levels of $\mathrm{Gd}^{3+}$ ions [20-22]. Furthermore, the linear relationship between $\mathrm{Gd}^{3+}$ concentration and line-width of the " $U$ " spectrum suggested that no concentration-dependent clustering effect was present, at least up to $x=0.04$ of $\mathrm{Gd}_{2} \mathrm{O}_{3}$ [22].

At $x=0.125$, the merging of these features into a single broad resonance ESR curve with $g_{\text {eff }} \approx 2$ suggests the prevailing of a strong interaction among Gd-Gd magnetic dipoles, perhaps grouped into clusters. On the other hand, pair distribution function (PDF) measurements are in agreement with $\mathrm{Gd}$ clusterization since the local scale of $\mathrm{Ce}_{1-x} \mathrm{Gd}_{x} \mathrm{O}_{2-x / 2}$ with $x<0.5$ compounds is described by a $\mathrm{Gd}_{2} \mathrm{O}_{3}$ droplets embedded in globally fluoritic sample matrix [4]. $\mathrm{Gd}^{3+}$ ion clusters were also introduced by [17] and investigated by Kliava et al. [23] to explain both the magnetization and ESR measurements of $\mathrm{Gd}_{2} \mathrm{O}_{3}$ doped glasses.

The steeper trend of $\Delta H_{p p}$ versus $x$ in the $\mathrm{CeO}_{2}$-like solid solution zone at $x \leq 0.3$ (see Figure 1) suggests that the added Gd ions perturb the Gd-Gd interaction stronger in 


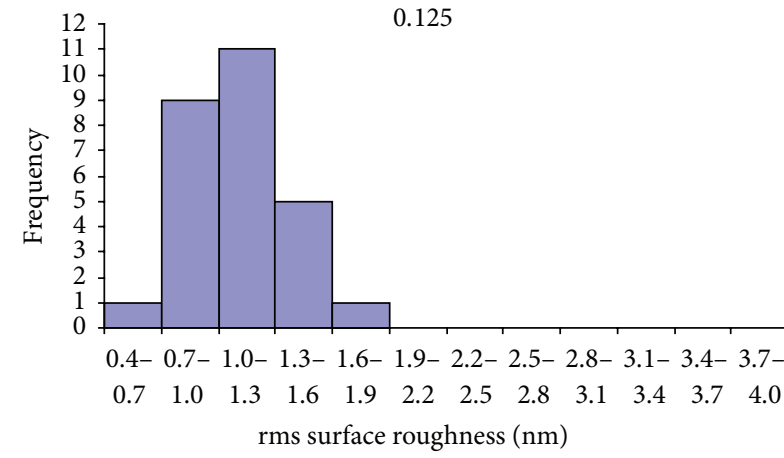

(a)

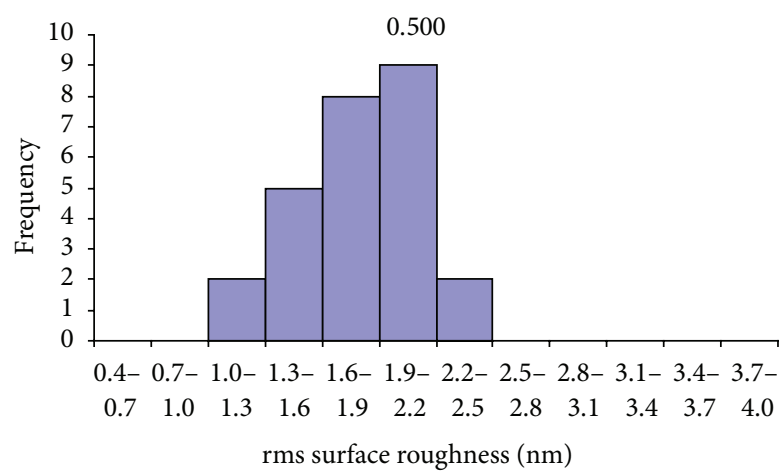

(c)

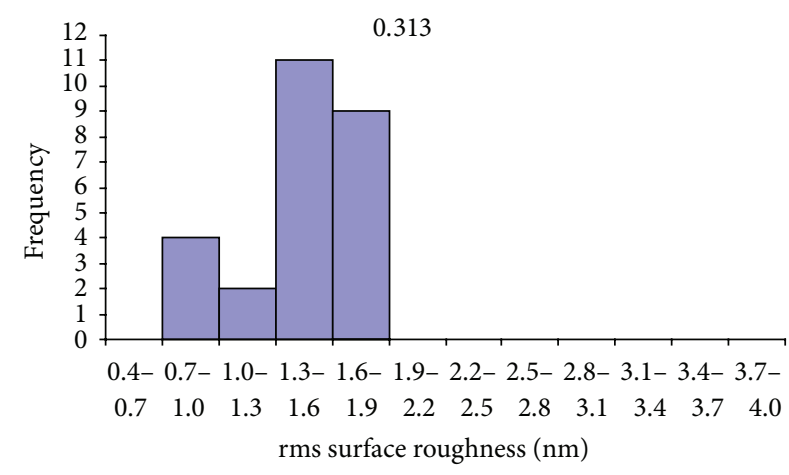

(b)

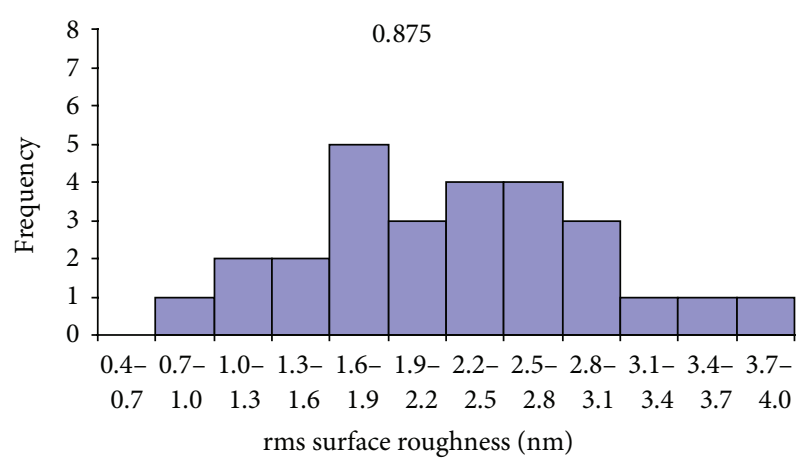

(d)

FIGURE 4: Histograms of the rms surface roughness values measured on $30 \times 30 \mathrm{~nm}^{2}$ areas and obtained analysing more than 20 AFM topography images.

the embedded $\mathrm{Gd}_{2} \mathrm{O}_{3}$ droplets than in the $\mathrm{Gd}_{2} \mathrm{O}_{3}$-like phase forming [4] at $x \geq 0.5$.

As stated above, ESR spectra are better described by Dysonian than single Lorentzian or Gaussian functions. It is interesting to investigate why the best fits of the ESR line profile were those obtained by a Dysonian function and why the last fits better at higher $x$ values (Figure 2). In most cases, a Dysonian line-shape appears when a sample becomes an electronic conductor [24-26]. However, it is well known that $\mathrm{Ce}_{1-x} \mathrm{Gd}_{x} \mathrm{O}_{2-x / 2}$ solid solutions have negligible electronic conductivity, apart from pure $\mathrm{CeO}_{2}$ and slightly Gd-doped compounds when annealed in reducing conditions $[2,11,27$, 28].

Besides, the Dysonian shape has been also attributed to the formation of surface polaritons. The last are propagating coupled electromagnetic-electric dipole excitation modes, created by the diffraction of the microwave ESR field on the surface roughness and are able to mix absorption and dispersion ESR modes, leading to a Dysonian spectral profile [29]. The formation of polaritons could be further favoured by the high spin-orbit interaction characterizing the surfacedoping Gd ions. Indeed, AFM data showed that the surface roughness increases and that its distribution broadens with samples characterized by higher $x$ values (Figures 3 and 4). This suggests that the Dysonian ESR profile observed with them and their surface roughness (inducing polaritons formation) are correlated to each other.
To summarize, we performed ESR and AFM measurements on $\mathrm{Ce}_{1-x} \mathrm{Gd}_{x} \mathrm{O}_{2-x / 2}$ as a function of $x$. In agreement with previous reports, for low Gd concentration $(x=0.02)$, the ESR signal is composed by three resonance lines at $g_{\text {eff }} \approx 2,2.8$, and 6 . For $x \geq 0.125$ a single broad signal is observed with a linewidth which increases upon doping. This $x$-dependent $\Delta H_{\mathrm{pp}}$ broadening is consistent with the increase of Gd-Gd interactions. Line-shape analysis reveals that the Dysonian function well describes all the spectra but the implication of electron delocalization disagrees with the compounds insulating ground state. By using AFM we observed an increase of surface roughness with increasing $x$. This gives an alternative explanation to support the Dysonian line-shape in $\mathrm{Ce}_{1-x} \mathrm{Gd}_{x} \mathrm{O}_{2-x / 2}$ system through surface polariton formation.

The present study also suggests that ESR spectroscopy can reveal not only a very useful technique to characterize sample paramagnetic impurities and defects, but also a quite sensitive tool to investigate the surface properties, like roughness, which may play an important role in the surface science and related applications.

\section{Conflict of Interests}

The authors declare that there is no conflict of interests regarding the publication of this paper. 


\section{References}

[1] B. C. H. Steel, "Dense ceramic ion conducting membranes," in Oxygen Ion and Mixed Conductors and Their Technological Applications, H. L. Tuller, J. Shoonman, and I. Riess, Eds., vol. 368 of NATO ASI Series E: Applied Sciences, pp. 323-345, 1997.

[2] Y.-M. Chiang, E. B. Lavik, and D. A. Blom, "Defect thermodynamics and electrical properties of nanocrystalline oxides: pure and doped $\mathrm{CeO}_{2}$," Nanostructured Materials, vol. 9, no. 1-8, pp. 633-642, 1997.

[3] M. Allieta, M. Brunelli, M. Coduri, M. Scavini, and C. Ferrero, "Differential pair distribution function applied to $\mathrm{Ce}_{1-x} \mathrm{Gd}_{x} \mathrm{O}_{2-x / 2}$ system," Zeitschrift für Kristallographie Proceedings, vol. 1, pp. 15-20, 2011.

[4] M. Scavini, M. Coduri, M. Allieta, M. Brunelli, and C. Ferrero, "Probing complex disorder in $\mathrm{Ce}_{1-x} \mathrm{Gd}_{x} \mathrm{O}_{2-x / 2}$ using the Pair Distribution Function Analysis," Chemistry of Materials, vol. 24, no. 7, pp. 1338-1345, 2012.

[5] M. Coduri, M. Scavini, M. Allieta, M. Brunelli, and C. Ferrero, "Local disorder in yttrium doped ceria $\left(\mathrm{Ce}_{1-x} \mathrm{Y}_{x} \mathrm{O}_{2-x / 2}\right)$ probed by joint X-ray and neutron powder diffraction," Journal of Physics: Conference Series, vol. 340, Article ID 012056, 2012.

[6] M. Coduri, M. Brunelli, M. Scavini et al., "Rare Earth doped ceria: a combined X-ray and neutron pair distribution function study," Zeitschrift für Kristallographie, vol. 227, no. 5, pp. 272279,2012

[7] M. Coduri, M. Scavini, M. Brunelli, and P. Masala, "In situ pair distribution function study on lanthanum doped ceria," Physical Chemistry Chemical Physics, vol. 15, no. 22, pp. 8495-8505, 2013.

[8] M. Coduri, M. Scavini, M. Allieta, M. Brunelli, and C. Ferrero, "Defect structure of Y-doped ceria on different length scales," Chemistry of Materials, vol. 25, no. 21, pp. 4278-4289, 2013.

[9] M. Coduri, M. Scavini, M. Brunelli, E. Pedrazzin, and P. Masala, "Structural characterization of Tb- and Pr-doped ceria," Solid State Ionics, vol. 268, pp. 150-155, 2014.

[10] M. Allieta, M. Scavini, L. Lo Presti et al., "Charge ordering transition in $\mathrm{GdBaCo}_{2} \mathrm{O}_{5}$ : evidence of reentrant behavior," Physical Review B-Condensed Matter and Materials Physics, vol. 88, no. 21, Article ID 214104, 2013.

[11] C. Oliva, M. Scavini, O. Ballabio, A. Sin, A. Zaopo, and Y. Dubitsky, "Percolative small-polarons conduction regime in $\mathrm{Ce}_{1-x} \mathrm{Gd}_{x} \mathrm{O}_{2-x / 2}$, probed by the EPR spectral intensity of $\mathrm{Gd}^{3+}$," Journal of Solid State Chemistry, vol. 177, no. 11, pp. 4104-4111, 2004.

[12] A. Sin, Y. Dubitsky, A. Zaopo et al., "Preparation and sintering of $C e_{1-x} G d_{x} \mathrm{O}_{2-x / 2}$ nanopowders and their electrochemical and EPR characterization," Solid State Ionics, vol. 175, no. 1-4, pp. 361-366, 2004

[13] C. Oliva, M. Allieta, M. Scavini, C. Biffi, I. Rossetti, and L. Forni, "Electron paramagnetic resonance analysis of $\mathrm{La}_{1-x} \mathrm{M}_{x} \mathrm{MnO}_{3+\delta}$ ( $\mathrm{M}=\mathrm{Ce}, \mathrm{Sr}$ ) perovskite-like nanostructured catalysts," Inorganic Chemistry, vol. 51, no. 15, pp. 8433-8440, 2012.

[14] M. Allieta, C. Oliva, M. Scavini, S. Cappelli, E. Pomjakushina, and V. Scagnoli, "Spin-lattice interaction in the insulatorto-metal transition of $\mathrm{GdBaCo}_{2} \mathrm{O}_{5}+\delta$," Physical Review BCondensed Matter and Materials Physics, vol. 84, no. 23, Article ID 235144, 2011.

[15] M. P. Pechini, "Method of preparing lead and alkaline earth titanates and niobates and coating method using the same to form a capacitor," U.S. patent 3330697, 1967.
[16] R. S. de Biasi and M. L. N. Grillo, "Electron spin resonance of diluted solid solutions of $\mathrm{Gd}_{2} \mathrm{O}_{3}$ in $\mathrm{CeO}_{2}$," Journal of Solid State Chemistry, vol. 178, no. 6, pp. 1973-1977, 2005.

[17] L. Cugunov and J. Kliava, "EPR of $\mathrm{Gd}^{3+}$ with $\mathrm{g}=6.0$ in glasses: a reinterpretation," Journal of Physics C: Solid State Physics, vol. 15, pp. L933-L936, 1982.

[18] H. J. A. Koopmans, M. M. A. Perik, B. Nieuwenhuijse, and P. J. Gellings, "The interpretation of broadened $\mathrm{Gd}^{3+} \mathrm{EPR}$ spectra of polycrystalline ceramics," Physica Status Solidi B: Basic Research, vol. 120, no. 2, pp. 745-751, 1983.

[19] C. Oliva, M. Scavini, S. Cappelli, M. Coduri, and M. Brunelli, "EPR investigation on nanosized superparamagnetic $\mathrm{Ce}_{1-(x+y)} \mathrm{Gd}_{x} \mathrm{Y}_{y} \mathrm{O}_{2-\delta}$ samples," in Proceedings of the 1st Joint Meeting ARPE-GERPE-GIRSE, Terrasini, Italy, October 2012.

[20] C. M. Brodbeck and L. E. Iton, "The EPR spectra of $\mathrm{Gd}^{3+}$ and $\mathrm{Eu}^{2+}$ in glassy systems," The Journal of Chemical Physics, vol. 83, no. 9, pp. 4285-4299, 1985.

[21] C. Legein, J. Y. Buzaré, G. Silly, and C. Jacoboni, “The local field distribution of $\mathrm{Gd}^{3+}$ in transition metal fluoride glasses investigated by electron paramagnetic resonance," Journal of Physics Condensed Matter, vol. 8, no. 23, pp. 4339-4350, 1996.

[22] C. B. Azzoni, D. Di Martino, A. Paleari, A. Speghini, and M. Bettinelli, "EPR study of $\mathrm{Gd}^{3+}$ doped lead oxide based glasses," Journal of Materials Science, vol. 34, no. 16, pp. 3931-3935, 1999.

[23] J. Kliava, A. Malakhovskii, I. Edelman et al., "Unusual magnetic transitions and nature of magnetic resonance spectra in oxide glasses containing gadolinium," Physical Review B-Condensed Matter and Materials Physics, vol. 71, no. 10, Article ID 104406, 2005.

[24] K. Sugawara and C. Y. Huang, "Electron paramagnetic resonance of $\mathrm{Gd}^{3+}$ in yttrium-group VA intermetallic compounds," Journal of the Physical Society of Japan, vol. 39, no. 3, pp. 643646, 1975.

[25] L. Bejjit and M. Haddad, "Oxygen content dependence of the EPR line shape in $\mathrm{GdBa}_{2} \mathrm{Cu}_{3} \mathrm{O}_{7}$-y single crystal," Applied Magnetic Resonance, vol. 22, no. 3, pp. 415-419, 2002.

[26] R. R. Urbano, P. G. Pagliuso, C. Rettori et al., "Gd ${ }^{3+}$ and $\mathrm{Eu}^{2+}$ local environment in $\mathrm{Ca}_{1-x} \mathrm{Eu}_{x} \mathrm{~B}_{6}(0.0001 \leq x \leq 0.30)$ and $\mathrm{Ca}_{1-x} \mathrm{Gd}_{x} \mathrm{~B}_{6}(0.0001 \leq x \leq 0.01)$, , Physica Status Solidi (A) Applications and Materials Science, vol. 203, no. 7, pp. 1550-1555, 2006.

[27] J. B. Goodenough, "Oxide-ion electrolytes," Annual Review of Materials Research, vol. 33, pp. 91-128, 2003.

[28] Y. M. Chiang, E. B. Lavik, I. Kosacki, H. L. Tuller, and J. Y. Ying, "Nonstoichiometry and electrical conductivity of nanocrystalline $\mathrm{CeO}_{2-x}$," Journal of Electroceramics, vol. 1, no. 1, pp. 7-14, 1997.

[29] J. I. Kaplan and J. Reuben, "Electron spin resonance line shapes of paramagnetic species on surfaces," The Journal of Physical Chemistry, vol. 86, no. 23, pp. 4465-4466, 1982. 

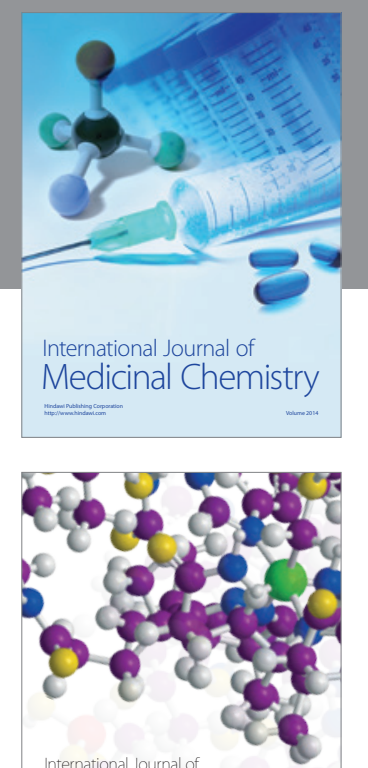

\section{Carbohydrate} Chemistry

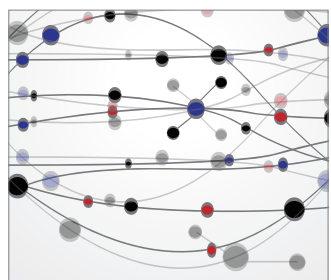

The Scientific World Journal
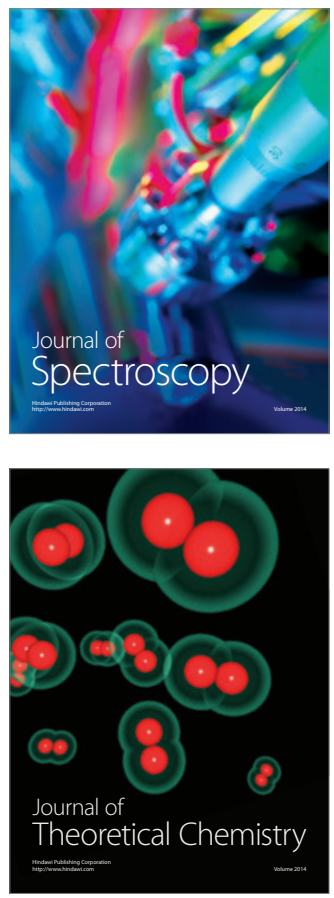
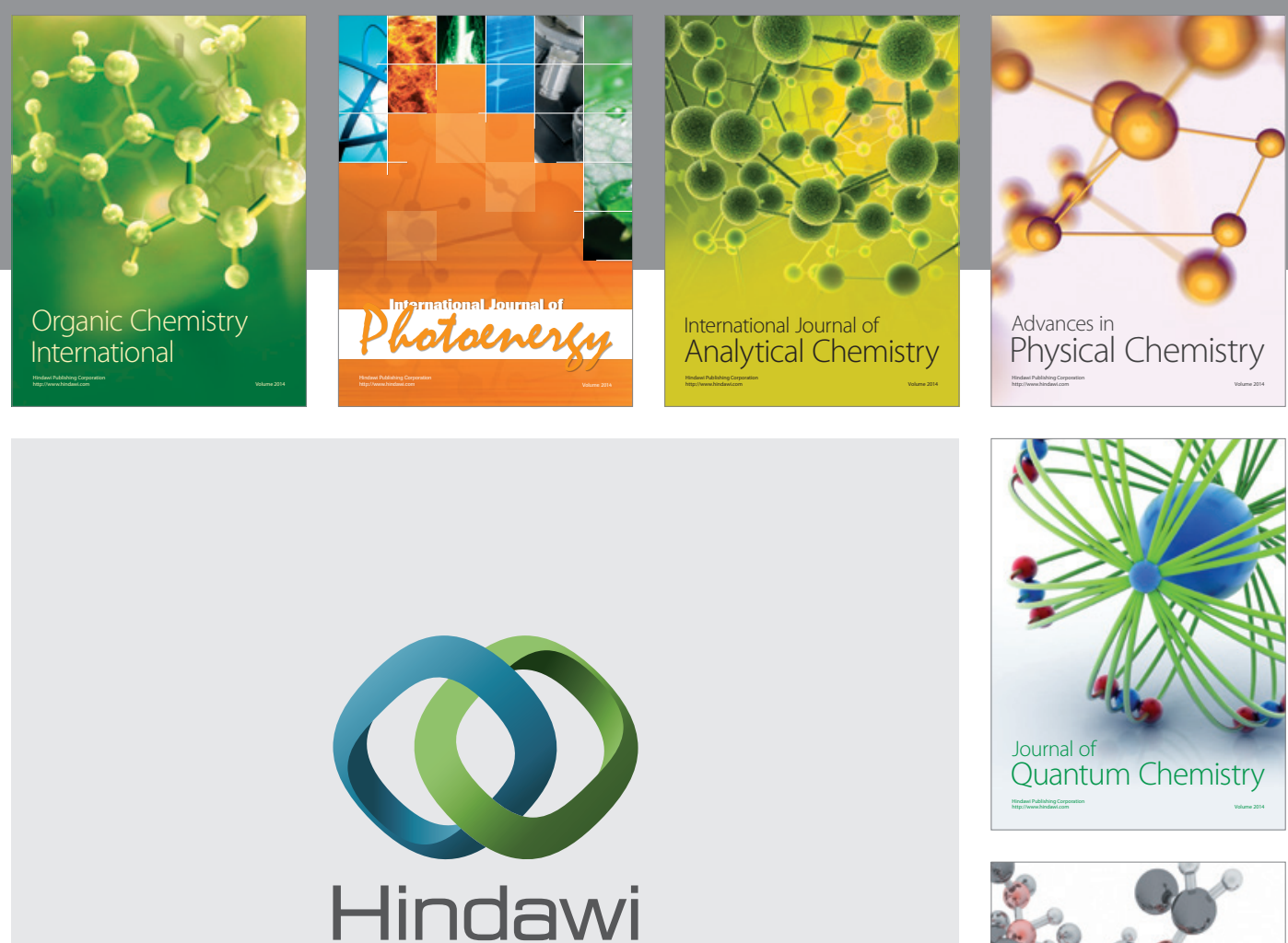

Submit your manuscripts at

http://www.hindawi.com

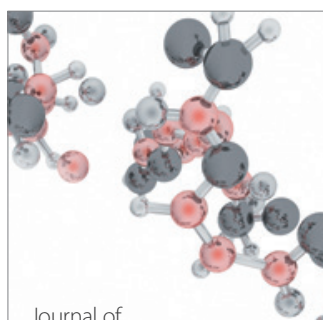

Analytical Methods

in Chemistry

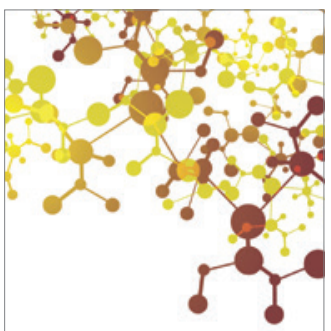

Journal of

Applied Chemistry

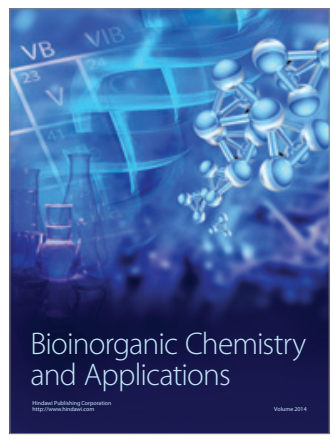

Inorganic Chemistry
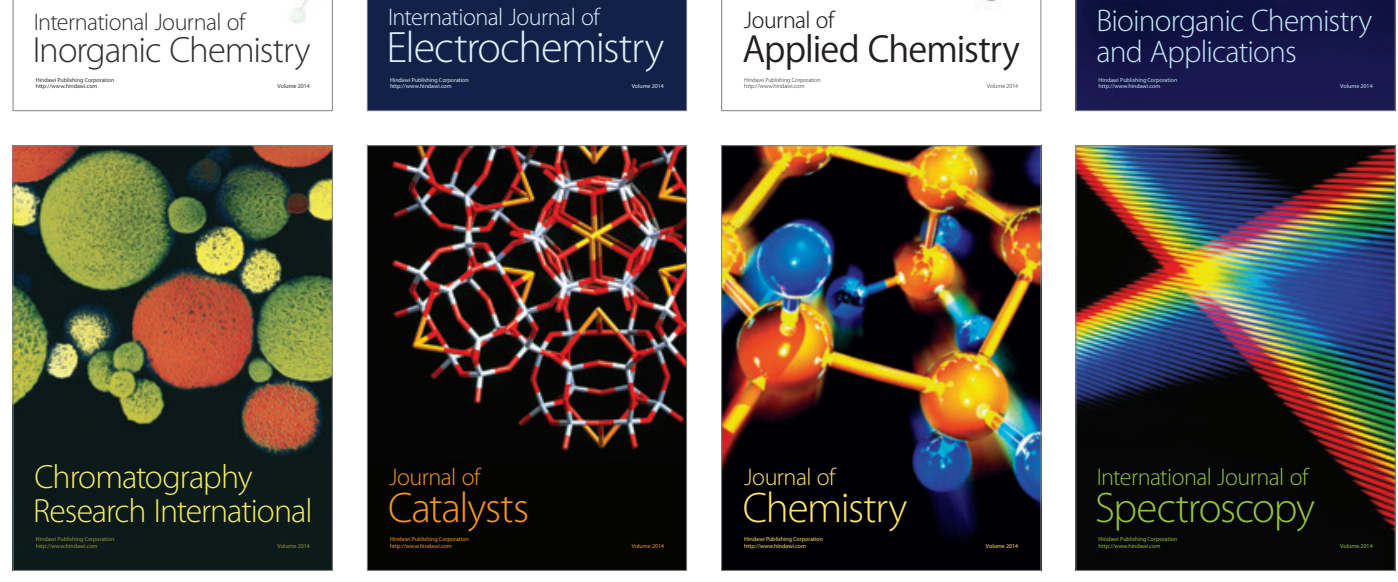